\title{
Article
}

\section{Comparison of the Local Order in Highly Oriented Pyrolitic Graphite and Bundles of Single-Wall Carbon Nanotubes by Nanoscale Extended Energy Loss Spectra}

P. Castrucci, F. Tombolini, M. Scarselli, C. Scilletta, M. De

Crescenzi, M. Diociaiuti, S. Casciardi, F. Rosei, and M. A. El Khakani

J. Phys. Chem. C, 2009, 113 (12), 4848-4855• DOI: 10.1021/jp810936c • Publication Date (Web): 26 February 2009

Downloaded from http://pubs.acs.org on March 20, 2009

\section{More About This Article}

Additional resources and features associated with this article are available within the HTML version:

- $\quad$ Supporting Information

- $\quad$ Access to high resolution figures

- $\quad$ Links to articles and content related to this article

- $\quad$ Copyright permission to reproduce figures and/or text from this article

\section{View the Full Text HTML}




\title{
Comparison of the Local Order in Highly Oriented Pyrolitic Graphite and Bundles of Single-Wall Carbon Nanotubes by Nanoscale Extended Energy Loss Spectra
}

\author{
P. Castrucci, ${ }^{*}$ F. Tombolini, M. Scarselli, C. Scilletta, ${ }^{\dagger}$ and M. De Crescenzi \\ Dipartimento di Fisica, Unità CNISM, Università di Roma "Tor Vergata", Via della Ricerca Scientifica 1, \\ 00133 Roma, Italy
}

\section{Diociaiuti}

Dipartimento di Tecnologie e Salute, Istituto Superiore di Sanità, 00161 Roma, Italy

\author{
S. Casciardi \\ Dipartimento di Igiene del Lavoro, ISPESL, 00040 Monte Porzio Catone, Italy
}

\author{
F. Rosei and M. A. El Khakani \\ Institut National de la Recherche Scientifique, INRS-Énergie, Matériaux et Télécommunications, 1650, \\ Bloulevard Lionel-Boulet, C.P. 1020, Varennes, Quebec, Canada J3X-1S2
}

Received: December 11, 2008; Revised Manuscript Received: January 21, 2009

\begin{abstract}
We compare the local order in single-wall carbon nanotubes (SWCNTs) and highly oriented pyrolitic graphite (HOPG) by means of nanoscale transmission extended energy loss fine structure (EXELFS) measurements above the carbon K edge. The HOPG EXELFS spectra and their Fourier transform were compared to their synchrotron extended X-ray absorption fine structure (EXAFS) counterpart and discussed within a multiple scattering framework. By comparing the experimental EXELFS data to spectra calculated using a theoretical model based on a single scattering approach, we showed the fundamental importance of considering multiple paths (involving up to eight body scattering) to reproduce the fine details of EXELFS features. Simulating EXELFS spectra of SWCNTs by our theoretical model is shown to represent a measure of their chiralities. Finally, the shrinkage of the nearest-neighbor distance in the Fourier transform observed for SWCNTs (absent in graphite) is interpreted within a simple model invoking anharmonic effects.
\end{abstract}

\section{Introduction}

Since their discovery, ${ }^{1-3}$ carbon nanotubes have attracted the attention of the scientific community, particularly because of their unique electronic properties that are intimately related to their structural helicity. In particular, the band structure of singlewall carbon nanotubes (SWCNTs) is entirely determined by the so-called chiral indices $(n, m)$ that characterize the rolling-up of the constituting graphene layer ${ }^{3}$ and uniquely determine the tube's electronic properties. Scanning tunnelling microscopy ${ }^{4,5}$ and selected area electron diffraction have been used to determine the chirality of individual and bundles of SWCNTs. ${ }^{6-8}$ Recently, by comparing the extended energy loss fine structure (EXELFS) spectra of a bundle of parallel SWCNTs, individual multiwall carbon nanotube (MWCNT), and free-standing highly oriented pyrolitic graphite (HOPG) thin flakes, we demonstrated that the carbon atoms in a single SWCNT are affected by an out-of-plane thermal motion greater than in MWCNTs or in HOPG structures. ${ }^{9}$ Identifying this important result was possible by recording spectra in a very wide energy range above the carbon $\mathrm{K}$ edge (i.e., EXELFS signals in a wavevector range up to $k=11 \AA^{-1}$ ).

* To whom correspondence should be addressed. Tel.: +39 0672594545. Fax: +3906 2023507. E-mail: castrucci@roma2.infn.it.

Also at Istituto dei Sistemi Complessi, CNR, 00016 Monterotondo Scalo, Italy.
Accessing a wider range of EXELFS oscillations also raises the challenging issue of their full theoretical reconstruction to achieve the most accurate analysis of the local order of the analyzed nanostructures. Here we compare an analysis of EXELFS spectra (covering a $k$ range of up to $11 \AA^{-1}$ ) of graphite and SWCNTs and their Fourier transforms in the context of a theoretical model which uses a full multiple scattering approach. By theoretically reproducing the experimental spectra and their Fourier transforms, we were able to achieve a better understanding of the scattering mechanisms in graphite and SWCNT nanostructures. Moreover, our analysis opens the possibility of the experimental labeling of the nanotubes by their respective chiralities given that EXELFS spectra can be acquired on single nanotubes. Finally, anharmonic effects observed in our earlier work on SWCNTs $^{9}$ are interpreted here in terms of the presence of an asymmetric radial distribution function, $g(R)$, induced by the lack of atoms in the direction perpendicular to the SWCNT surface. This interpretation also explains the temperature-induced Raman scattering shift reported in the breathing modes and the G-band of SWCNTs, in comparison with HOPG. ${ }^{10,11}$

\section{Experimental Methods}

II.a. SWCNT Synthesis. SWCNTs were synthesized by ablating a CoNi-doped graphite target, using a pulsed Nd:YAG laser in the superimposed double-pulse configuration. The target, placed in a quartz tube at the center of a furnace, was ablated with a laser intensity of $\sim 2.5 \times 10^{9} \mathrm{~W} / \mathrm{cm}^{2}$, at a temperature 
of $\sim 1100{ }^{\circ} \mathrm{C}$ in a flowing $\mathrm{Ar}$ gas. The synthesis product was collected on a water-cooled surface at the furnace exit. More details on the SWCNTs synthesis can be found elsewhere. ${ }^{12}$ Raman spectra of the as-grown SWCNTs have revealed that the nanotubes have a narrow diameter distribution peaking around $1.2 \pm 0.1 \mathrm{~nm}$ (their radial breathing mode is centered around $\left.185 \mathrm{~cm}^{-1}\right){ }^{12}$

II.b. Transmission Electron Microscopy Analysis. Transmission electron microscopy (TEM) experiments were performed with an FEI TECNAI 12 G2 Twin operated at an accelerating voltage of $120 \mathrm{kV}$, equipped with an electron energy filter (Gatan Image Filter, BioFilter model) and a Peltier-cooled charge-coupled device based slow scan camera (Gatan multiscan camera, model 794). The energy resolution of the spectra was estimated to be about $0.8 \mathrm{eV}$ by considering the full width at half-maximum of the elastic electron peak. Thus, all the reported features in the EXELFS spectra are not affected by our instrumental energy resolution.

The as-grown SWCNTs were diluted in isopropyl alcohol, quickly sonicated, and a drop of the resulting solution was used to disperse the nanotubes on a gold TEM grid (mesh 1000) without using any supporting amorphous carbon film. Bundles of parallel SWCNTs were found to be in a free-standing configuration (i.e., bridging adjacent grid wires). All the EXELFS measurements were performed on a single bundle of free-standing tubes. Similar experiments were also carried out on tiny flakes of HOPG, sufficiently thin $(\sim 20 \mathrm{~nm})$ to allow high electron transmission and avoid multiple scattering processes inducing multiple plasmon losses that mask the genuine energy loss fine structures. ${ }^{13}$ The use of such a thin film avoided the deconvolution procedure of the plasmon losses close to the elastic electron peak. The EXELFS spectra were acquired above the carbon $\mathrm{K}$ edge by probing a sample region of $\sim 50 \mathrm{~nm} \times$ $50 \mathrm{~nm}$ selected in the imaging mode.

TEM images were acquired before and after each electron energy loss spectroscopy (EELS) measurement to ensure that the area under investigation had not suffered any morphological change and/or damage that may arise from its long exposure to the high-energy electron beam. As a matter of fact, if the thermal heat is not dissipated efficiently, the nanotubes under investigation appear to change their morphology and even break sometimes. This depends on many factors, such as their bundle diameter, how much the connections to other carbon nanotubes (CNTs) and/or to the gold TEM grid are able to ensure a good heat transfer, and the beam current density. Various bundles of SWCNTs (with diameters down to $\sim 5 \mathrm{~nm}$ ) were successfully analyzed by this procedure. However, EXELFS spectra with satisfactory signal-to-noise ratio were quite challenging to obtain on single nanotubes. This difficulty is mainly due to the damaging particularly observed on single tubes after their long exposure to the electron beam (at least $30 \mathrm{~min}$ is required for collecting a whole spectrum with an energy range about 500 $\mathrm{eV}$ wide). On the other hand, near-edge EELS ( $\sim 50 \mathrm{eV}$ above the ionization edge) spectra on single isolated tubes were recorded without sizable and visible damaging problems. ${ }^{14}$ Moreover, near-edge EELS was also used to ensure that no Ni/ Co catalyst traces are present in the investigated carbon nanostructures. To this end, EELS spectra were systematically recorded, with suitable energy range (i.e., 870-852 and $793-778 \mathrm{eV}$, respectively), to detect the possible presence of any features of the $\mathrm{Ni}$ and $\mathrm{Co}_{2,3}$ edges. Thus, one can detect the presence of $\mathrm{Ni}$ and $\mathrm{Co}$ atoms, if there is any, as long as they are not under the detection limit of five atoms over $10^{3}$. Likewise, EELS measurements (around the $\mathrm{K}$ edges of $\mathrm{O}$ and
$\mathrm{N})$ confirmed the absence, within the detection limit, of any oxygen or nitrogen impurities that might contaminate the surface of nanotubes or HOPG. The detection limit depends on the number of atoms measured, the shape of the edge, and the signal-to-noise ratio. In our specific case, the number of carbon atoms in the illuminated area of $50 \mathrm{~nm} \times 50 \mathrm{~nm}$ amounts to about $10^{5}$ atoms. Consequently, our detection limit was estimated to be of about $5 \times 10^{-3}$, according to the pioneering work of Colliex. ${ }^{15}$

II.c. EXELFS Data Analysis and Their Comparison with EXAFS. Extended X-ray absorption fine structure (EXAFS) ${ }^{16-18}$ is a powerful technique for probing the local order around a selected absorbing atom. The extended fine structure above a core photoabsorption edge (typically a $\mathrm{K}$ edge) is interpreted in terms of the interference between the outgoing excited photoelectron wave function and its scattered part by the atoms surrounding the absorber. The EXAFS signal analysis gives direct access to the local geometry around the absorbing atom in terms of the number of its nearest neighboring atoms $\left(N_{j}\right)$ of type $j$ located at distance $R_{j}$ from it.

The use of high-energy electrons in EXELFS spectroscopy to detect the fine structure losses above a core level has been demonstrated by Ritsko et al. ${ }^{19}$ to be a highly attractive alternative to X-rays. Thus, EXELFS measurements are typically carried out by recording the inelastic scattered electrons from a self-supported thin film using a standard TEM. The measured transition in EXELFS has variable energy amplitude, whereas the exciting electron energy remains strictly fixed. ${ }^{20}$ This allows to measure the number of scattered electrons of the primary beam which have excited core electrons of the medium to the same interference final state of the X-ray absorption process. EXELFS has an unprecedented nanoscale spatial resolution, permitting the localized study of the chemical nature and local ordering in the area exposed to the electron beam spot. ${ }^{21}$

Analysis of EXAFS-like signals of low- $Z$ absorbers (e.g., C, $\mathrm{N}$, and $\mathrm{O}$ ) involving low- $Z$ atom-scatterers presents a number of difficulties because EXAFS oscillations are fewer and less intense than for the high- $Z$ atoms. This occurs because light element bonds are very short and their scattering amplitude rapidly decreases above the absorption edge. HOPG has been investigated by both EXAFS ${ }^{22-25}$ and EXELFS. ${ }^{26,27}$ Signals have been analyzed by taking into account only the backscattering of the excited core electrons by the nearest-neighbor atoms (i.e., single scattering regime) and neglecting any other scattering path involving more than two atoms. Although such analyses ${ }^{22-27}$ yield the first-shell coordination numbers and their corresponding $\mathrm{C}-\mathrm{C}$ bond lengths, they have definitely failed in reproducing the EXAFS-like signal in the entire $k$ wavevector range and particularly for $k$ values $<6 \AA^{-1}$. On the other hand, experimental energy loss spectra above the carbon $\mathrm{K}$ edge reported so far have been acquired over a very narrow energy window $\left(E_{\max }\right.$ about $550 \mathrm{eV}$ ), thereby limiting the valuable information that can be extracted from the EXELFS oscillations. ${ }^{26,27}$

Within the limit of the dipole approximation, EXELFS features are very similar in nature to the EXAFS ones (obtained by synchrotron radiation excitation), and therefore EXELFS spectra can be analyzed with the EXAFS data procedure. ${ }^{16,17}$ An evaluation of the electron scattering cross section for EXELFS can be made within the framework of the Born approximation. ${ }^{28}$ In the limit of $\mathbf{q} \cdot \boldsymbol{r}_{\mathrm{a}} \ll 1$ (dipole approximation) the electron yield distribution $N(E)$ of the measured scattered electrons is proportional to the differential cross section of the momentum transferred $\mathbf{q}$ to the excited electron by the primary 
electron beam, depending on the experimental scattering geometry ${ }^{29,30}$

$$
N(E)=\mathrm{d} \sigma / \mathrm{d} q \propto q^{-1}\left|\left\langle\psi_{\mathrm{f}}\left|\boldsymbol{\varepsilon}_{\mathrm{q}} \cdot \boldsymbol{r}_{\mathrm{a}}\right| \psi_{\mathrm{i}}\right\rangle\right|^{2}
$$

where $\boldsymbol{\varepsilon}_{\mathrm{q}}$ is the unit vector in the direction of the $\mathbf{q}$ vector and $\boldsymbol{r}_{\mathrm{a}}$ is position vector of the core electron involved in the transition. $\left|\Psi_{\mathrm{i}}\right\rangle$ and $\left|\Psi_{\mathrm{f}}\right\rangle$ are the electron wave functions describing the initial state and excited final state of the core electron, respectively. The formula above is very similar to the matrix element which appears in the X-ray absorption coefficient: ${ }^{16,17}$

$$
\chi(E) \propto\left|\left\langle\psi_{\mathrm{f}}\left|\boldsymbol{\varepsilon} \boldsymbol{r}_{\mathrm{a}}\right| \psi_{\mathrm{i}}\right\rangle\right|^{2}
$$

where $\varepsilon$ is the unit vector of the electric field in the direction of the X-ray polarization.

The structures superimposed on the isolated atomic contribution originate in the final state $\left|\Psi_{\mathrm{f}}\right\rangle$ which can be written as a sum of the outgoing spherical wave from the absorbing atom and its back-reflected portion from the surrounding atoms. This description of the final state produces the same features for both $N(E)$ and $\chi(E)$ resulting in a superposition of several sinusoidallike functions each containing the structural local parameters around the absorbing atom. ${ }^{16}$

To evaluate the validity of the dipole approximation around the carbon $\mathrm{K}$ edge $(\Delta E=284.6 \mathrm{eV})$, for a primary electron beam with an energy $E_{\mathrm{p}}$ of $120 \mathrm{keV}$, it is possible to estimate the minimum $\left(q_{\mathrm{min}}\right)$ momentum transferred to the $1 \mathrm{~s}$ core electron. ${ }^{13}$

$$
q_{\min }=\left(2 m / \hbar^{2}\right)^{1 / 2}\left(E_{\mathrm{p}}^{1 / 2}-\left(E_{\mathrm{p}}-\Delta E\right)^{1 / 2}\right)
$$

With the above-mentioned parameters, the minimum momentum transferred is $0.21 \AA^{-1}$. Thus, for $r_{\mathrm{a}}=0.089 \AA$ (the radius of the carbon $1 \mathrm{~s}$ shell), we obtain $\mathbf{q} \cdot \boldsymbol{r}_{\mathrm{a}}=0.019(\ll 1)$, demonstrating thereby the validity of the dipole approximation for our geometrical experimental setup. As a consequence, experimental EXELFS spectra can be analyzed following the standard procedure used to extract the oscillating EXAFS features above the absorption edges. ${ }^{16,17}$ The onset of the ionization edges has been located at the inflection point of the $\pi^{*}$ features corresponding to the position of the Fermi level at $284.6 \mathrm{eV}$. After background subtraction and edge step normalization of the absorption data, the $\chi(k)$ signals were extracted over a range extending up to about $11.0 \AA^{-1}$ ( $k$ being the wavevector of the core electron ionized above the Fermi level). The structural analysis was carried out through Fourier transformation (FT) of the extracted $k \chi(k)$ signals (the FT window was chosen to extend from about $k_{\min }=4.4 \AA^{-1}$ to $k_{\max }=11.0 \AA^{-1}$ ).

We simulated the EXELFS data by using the FEFF8.2 software that calculates the extended X-ray absorption fine structure. ${ }^{31}$ Simulations of the EXELFS region were performed for HOPG and for SWCNTs with a diameter of about $1.2 \mathrm{~nm}$ (corresponding to various $(n, m)$ chiralities), following several steps. First, we prepare the atomic coordinates for the structure under study; we choose the absorbing atom, and set the radius of the spherical cluster around it containing all the atoms to be considered as scatterers. Then, the scattering potentials are computed using a self-consistent procedure starting from the Mattheiss prescription in the initial iteration ${ }^{32}$ and considering the Hedin-Lundqvist expression for the exchange part of the potential $^{33}$ and an edge shift of $-2 \mathrm{eV}$ from the Fermi level. This self-consistent iteration has been applied to a cluster of 4 $\AA$ around the central $\mathrm{C}$ atom (higher cluster radii did not lead to any appreciable change of the self-consistent scattering

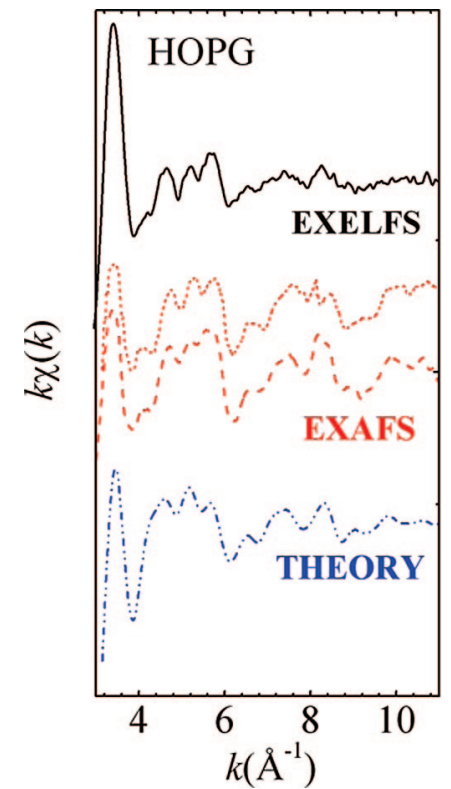

Figure 1. HOPG $k \chi(k)$ signals measured through EXELFS (solid black line), EXAFS recorded at the magic angle and with the $\mathbf{E}$ vector of X-ray parallel to the HOPG ring planes (short-dashed and dashed red lines, respectively), and that calculated (dot-dashed blue line) within a multiple scattering approach with a cluster radius of $8 \AA$ around the central atom and taking into account contributions to the $k \chi(k)$ oscillations of couples, triplets, quadruplets,..., up to octets of atoms beginning and ending at the central atom. EXAFS oscillations were adapted with permission from Figure 10 of ref 25, copyright 2007 American Physical Society, and from Figure 1 of ref 23, copyright 1988 American Physical Society, respectively.

potentials). In this way, 30 carbon atoms were introduced into the computation. Subsequently, the scattering phase shifts (both the absorbing and backscattering carbon atoms), dipole matrix elements, X-ray cross section, and angular momentum projected density of states were calculated. The leading multiple scattering paths for the cluster are enumerated (according to their effective distance from the central atom and the scattering amplitudes), and their degeneration degree is calculated. The EXAFS parameters from one or more paths are combined to calculate the total EXAFS spectrum. The code uses a constructive algorithm with several path importance filters to explore all significant multiple scattering paths in order of increasing path length and the required number of scattering atoms. An efficient degeneracy checker is used to identify equivalent paths (based on similar geometry, path reversal symmetry, and space inversion symmetry). Since the number of multiple scattering paths quickly becomes large, it is necessary to eliminate as many paths as possible. To limit the number of paths under consideration, we eliminated all the paths whose intensity was lower than $3 \%$ and $4 \%$ with respect to that of the first neighbor scattering, respectively, when the code rapidly finds the paths using a plane-wave approximation and when it actually computes the paths' amplitude within the full curved wave calculation. Finally we have assumed the amplitude reduction factor contained in the EXAFS formula, $S_{0}{ }^{2}$, equal to one and for every path, we have used the same harmonic Debye-Waller factor of $0.003 \AA^{2}{ }^{34}$

\section{Results and Discussion}

III.a. EXELFS Analysis of HOPG. Figure 1 compares the HOPG $k \chi(k)$ signals measured through EXELFS (solid black line) and those obtained with X-ray absorption recorded at the 

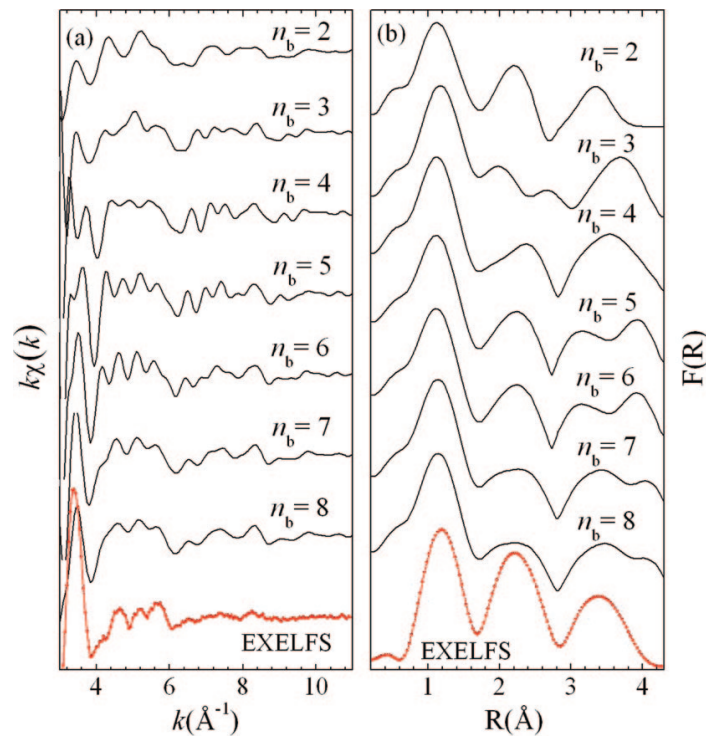

Figure 2. (a) HOPG $k \chi(k)$ simulated signals with a cluster radius fixed at $R=8.0 \AA$ and considering the contributions of all the multiple scattering of $n_{\mathrm{b}}$-body paths (with $n_{\mathrm{b}}$ varying from 2 (i.e., single scattering) to 8). The experimental EXELFS $k \chi(k)$ oscillations of HOPG (dotted red line) are included. The $R$ factor values for the average among the best $S_{0}{ }^{2}$ values corresponding to each calculated EXELFS $k \chi(k)$ signal are reported in the text. (b) The Fourier transforms signals $(F(R))$ of the oscillations shown in panel a. The $k$ range used to calculate the $F(R)$ curves extends from about $k_{\min }=4.4 \AA^{-1}$ to $k_{\max }=11 \AA^{-1}$.

magic angle so as to have an isotropic average spectrum irrespective of the degree of texture ${ }^{35}$ and with the $\mathbf{E}$ vector of the X-rays parallel to the HOPG ring planes, as reported by Diaz et al. ${ }^{25}$ (short-dashed red line) and by Comelli et al. ${ }^{23}$ (dashed red line), respectively. Slight differences can be observed between these two spectra due to the different polarizations, thus meaning that the contribution of the out-ofplane carbon atoms is less important than the ones in-plane. The dotted blue lines are the best theoretical simulations of the HOPG spherically averaged spectra (dotted blue lines), as computed with a full multiple scattering approach for a cluster of $8 \AA$ around the ionized atom.

As expected within the frame of the dipole approximation, the EXELFS experimental signal is in good agreement with $\mathrm{X}$-ray absorption spectra over the whole wavevector and intensity ranges. ${ }^{23,25}$ The detailed procedure used to obtain the results displayed in Figure 1 will be briefly discussed hereafter. Parts a and $\mathrm{b}$ of Figure 2 show the simulations of the $k \chi(k)$ oscillations of HOPG and their corresponding FTs (i.e., $F(R)$ ) calculated for a cluster of $8 \AA$ around the central atom as a function of increasing number, $n_{\mathrm{b}}$, of atoms involved in the scattering (i.e., each spectrum is made of the sum of all $n_{\mathrm{b}}$ body paths contributions ${ }^{36}$ ). For comparison purposes, the measured EXELFS oscillations and their corresponding $F(R)$ curve (red dotted lines) are also included in Figure 2. The agreement between theory and experiment is quantified by the value of the $R$ factor, defined as the sum of the absolute values of the differences between theory and experiment normalized by the number of experimental points. The intensities of the simulated curves have been multiplied by a factor that has been varied between 0.1 and 1 to account a posteriori for an amplitude reduction factor $S_{0}^{2}$ not equal to one. The minimum $R$ has been always obtained when eight-body paths $\left(n_{\mathrm{b}}=8\right)$ have been considered. Moreover, the lowest $R$ factor values were found to occur for $S_{0}^{2}$ values ranging between 0.1 and 0.3 . In particular, the $R$ factor values for the average among the best $S_{0}^{2}$ values

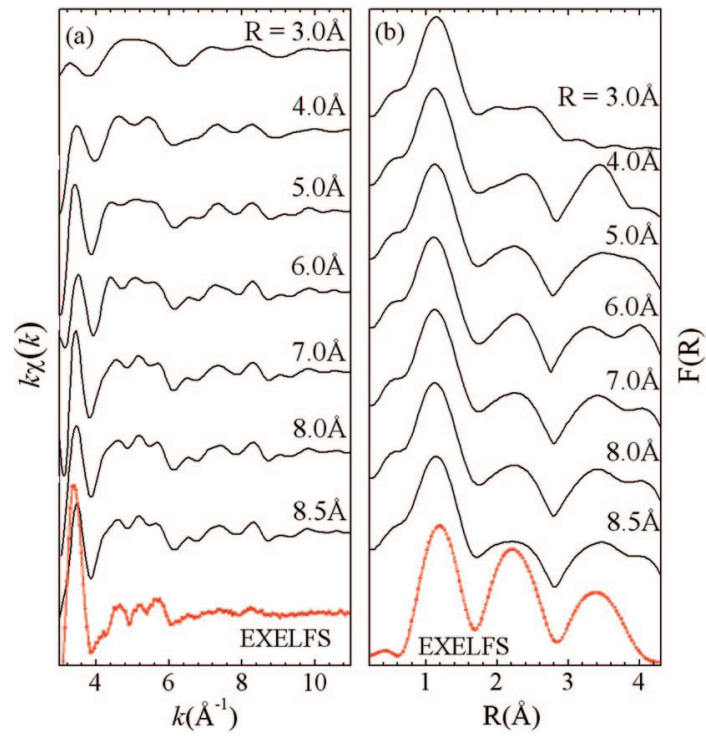

Figure 3. HOPG $k \chi(k)$ simulated signals calculated for increasing cluster radii from 3.0 to $8.5 \AA$ and for paths contributions of up to $n_{\mathrm{b}}$ $=8$ (a) and their corresponding Fourier transform curves (b). The $k$ range used to calculate the $F(R)$ curves extends from about $k_{\min }=4.4$ $\AA^{-1}$ to $k_{\max }=11 \AA^{-1}$. The experimental EXELFS $k \chi(k)$ oscillations of HOPG (a) and their corresponding $F(R)$ curve (b) are also included (dotted red line). The $R$ factor values for the average among the best $S_{0}{ }^{2}$ values are $0.0136,0.0123,0.0120,0.0124,0.0123,0.0119,0.0119$ for each cluster radius from $R=3$ to $8.5 \AA$, respectively.

resulted to be $0.0145,0.0147,0.0123,0.0122,0.0123,0.0126$, 0.0119 for each calculated signal from $n_{\mathrm{b}}=2$ to 8 , respectively. We also note in Figure $2 b$ that the multiple scattering contributions do not affect the nearest-neighbor position (always located at $1.14 \AA$ ), whereas the intensity and position of farther neighbors peaks are dramatically changed by the $n_{\mathrm{b}}$ value (i.e., they involve several body paths).

The HOPG theoretical spectra were obtained by averaging the two EXELFS spectra calculated by considering as central atoms the two basis atoms of the unbuckled hexagonal unit cell $\left(\mathrm{PG}_{3} / \mathrm{mmc}\right.$ space group) with lattice parameters $a=2.456 \AA$ and $c=6.696 \AA .{ }^{37}$ The radius of the cluster, containing all the scatterers around the central one, has been chosen to be $8 \AA$ as no changes were observed in the calculated $\chi(k)$ signals for higher radii. Figure 3 a shows how the radius of the cluster acts on the resulting $k \chi(k)$ signal calculated for $n_{\mathrm{b}}=8$. In particular, we considered radii from 3 to $8.5 \AA$, and this means that we included from 12 to 298 (namely, 296 for one ionization site and 298 for the other) atoms around the central one. Their corresponding FT (i.e., $F(R))$ curves are reported in Figure $3 \mathrm{~b}$. A cluster of radius equal to $3 \AA$ contains only carbon atoms in the first three shells on the basal plane, and this is why the corresponding $F(R)$ shows only three features for radial distances less than $3 \AA$. The simulation is stopped for a cluster radius of $8.0 \AA$ when no further improvement of the $R$ factor has been obtained in $k$ space between simulated and experimental HOPG spectra (see caption of Figure 3a). Again, an $S_{0}^{2}$ value between 0.1 and 0.3 gives the lowest $R$ factor values.

Also in this case, the increasing number of atoms plays an important role in reproducing the experimental oscillations. It is worth noting that no sizable differences in the spectra can be appreciated passing from a radius of $8 \AA$ to one of $8.5 \AA$. We considered thus that convergence is reached for a radius value of $8 \AA$. Such rather high values of the convergence radius and of $n_{\mathrm{b}}$ resulted to be a peculiarity of graphite. In fact, because of the short $\mathrm{C}-\mathrm{C}$ distances in a graphite lattice, the excited 


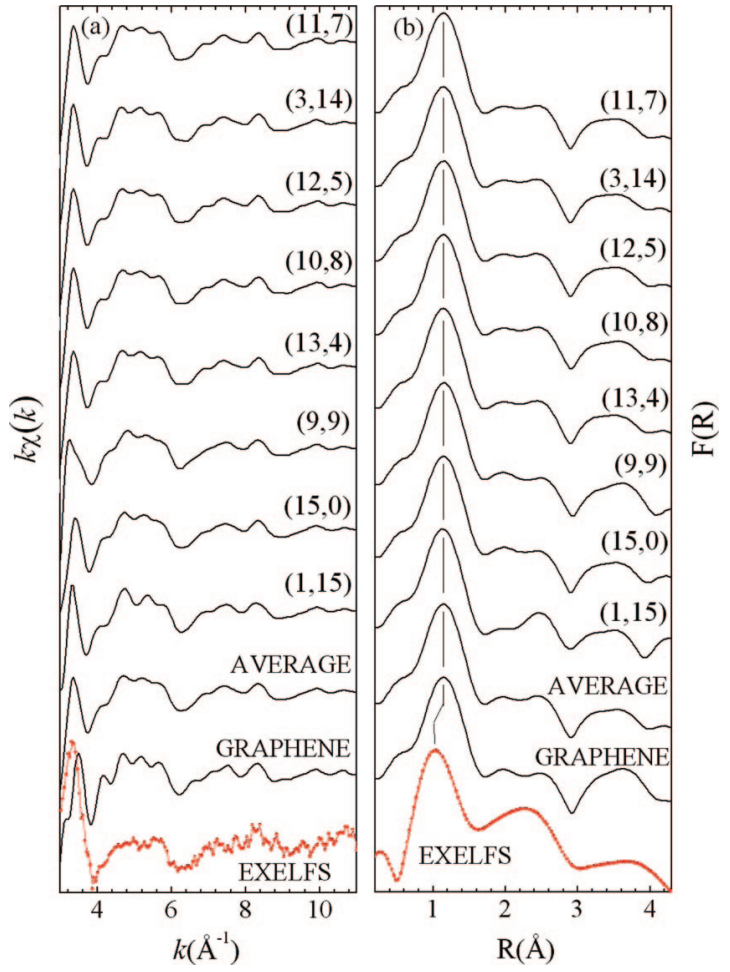

Figure 4. (a) $k \chi(k)$ simulated signals for a graphene layer and for SWCNTs having a diameter of $\sim 1.2 \mathrm{~nm}$ but with different $(n, m)$ helicities. All the simulations were performed by fixing the cluster radius to $8 \AA$ and accounting for all the multiple scattering paths contributions of up to $n_{\mathrm{b}}=8$. The calculated $k \chi(k)$ signals of the various chiralities were summed up, and an averaged resulting signal is also included together with the experimental EXELFS data recorded on a bundle of parallel SWCNTs of $22 \pm 2 \mathrm{~nm}$ diameter; (b) their corresponding Fourier transform curves calculated using a $k$ range extending from about $k_{\min }=4.4 \AA^{-1}$ to $k_{\max }=11 \AA^{-1}$. Note the sizable shift of 0.15 $\AA$ in the first nearest neighbor of the experimental $F(R)$ compared to all the other calculated $F(R)$ curves.

electron, on its mean free path, will interact with a rather large number of atoms compared to other elements with longer lattice parameter.

III.b. EXELFS Analysis of SWCNTs. On the basis of the previous results we have considered the HOPG data as a reference to simulate the single carbon nanotubes $\chi(k)$ signal. SWCNTs can be considered to be formed by rolling up a single layer of graphite where the so-called chirality vector, $\mathbf{C}_{\mathrm{h}}$, is defined by $\mathbf{C}_{\mathrm{h}}=n \hat{\mathbf{a}}_{1}+m \hat{\mathbf{a}}_{2}\left(\hat{\mathbf{a}}_{1}\right.$ and $\hat{\mathbf{a}}_{2}$ are the unit vectors in the two-dimensional hexagonal lattice, and $n$ and $m$ are integers). Thus, the chirality vector defines the nanotube's circumference, and different values of $n$ and $m$ lead to different nanotube structures. To reconstruct theoretically the EXELFS signal, we used a cluster radius around the central carbon atom of $8.0 \AA$, all the multiple scattering paths contribution up to $n_{\mathrm{b}}=8$, and a Debye-Waller factor of $0.003 \AA^{2}$ for each path. The experimental EXELFS $k \chi(k)$ signal acquired on a $22 \mathrm{~nm}$ diameter bundle of SWCNTs is shown at the bottom of Figure 4a (dotted red line). TEM images of this rope, reported in ref 9 , allowed us to measure directly the diameter of the nanotubes, which was found to be $\sim 1.2 \mathrm{~nm}$. This measurement corroborates well with our previous analyses of the radial breathing mode frequencies in the Raman spectra of these laser-grown SWCNTs. ${ }^{12}$ From these Raman analyses, more than $90 \%$ of the SWCNTs, grown under the above-described experimental conditions, have nanotube diameters in the $1.2-1.3 \mathrm{~nm}$ range, whereas the remaining $8 \%$ possess diameters of around $1.4 \mathrm{~nm} .^{12}$

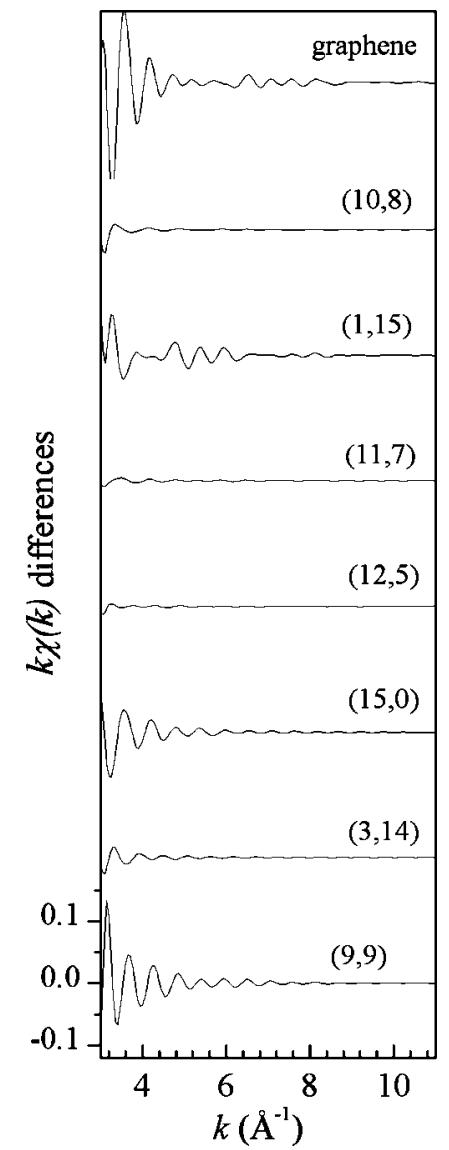

Figure 5. Differences between the EXELFS theoretical calculation of each chirality SWCNTs reported in Figure 4a with one of them (e.g., the $(13,4)$ in the present case). The $y$-scale is the same for all the differences as that shown in the bottom figure.

More importantly, even if some other tube diameters may be present inside the bundles, their percentage is too low and consequently their contribution to the EXELFS oscillations would be negligible (a point confirmed by the results discussed hereafter). Therefore, to rebuild theoretically these EXELFS oscillations, we considered that the bundle is formed by $\sim 1.2$ $\mathrm{nm}$ diameter nanotubes with different chiralities. Since SWCNTs are known to grow semiconducting for $66 \%$ and metal for $33 \%$, we assume that a similar statistical weight is maintained in the bundle. We thus computed the $k \chi(k)$ oscillations for several $(n$, $m$ ) SWCNTs having a diameter around $1.2 \mathrm{~nm}$. In particular, calculations have been performed for the following chiralities: $(15,0),(13,4),(9,9),(12,5),(10,8),(3,14),(11,7),(1,15)$ (the former three giving rise to metal nanotubes) using the CNTs coordinates computed by means of the application of ref 38 . The obtained results are shown in Figure 4a. Moreover, the $k \chi(k)$ oscillations simulated for a flat graphene sheet are also reported in Figure 4a. A few differences (in position and intensity) especially in the low- $k$ range (mainly from 3 to $6 \AA^{-1}$ ) can be observed between graphene and nanotubes with different chiralities, evidencing thereby the effect of tube curvature that affects the local structure around each carbon atom. On the other hand, we found that the $k \chi(k)$ signals of $(15,0),(9,9)$, and (1, 15) nanotubes show a few differences in terms of peaks position and intensity with respect to all the other chiralities considered (this is also translated in their corresponding FT curves reported in Figure 4b). These chiralities correspond to a higher order in the carbon atoms positions around the central one in the nanotube network when compared to others. This is more evident for the farthest neighbors thus reflecting mainly in the 
low- $k$ range $k \chi(k)$ oscillations. To demonstrate that this technique is able to evaluate the chirality of an isolated SWCNT we calculated the differences between one chirality (e.g., (13, 4)) and all the others (Figure 5). We verified that this difference is sizable in shape and it is of the order of $10^{-1}-10^{-2}$. This should require a very good signal-to-noise ratio of the experimental signal in order to be detected. Nevertheless, the position of the first peak in the $F(R)$, corresponding to the first coordination shell, is found to be insensitive to nanotube chirality. The chirality-associated changes are rather observable for the other features that are affected by the presence of the curvature modifying both the atomic position and the multiple scattering paths. Since there is no reason to consider any of the chiralities that might form the rope as dominant, we simply averaged all the simulated $k \chi(k)$ signals and compared it to the experimental data (Figure 4a) (a similar comparison for $F(R)$ is reported in Figure 4b). Interestingly, despite the quite good agreement between the measured EXELFS and the calculated spectra, the first peak of the Fourier transform for the experimental curves appears to be slightly shifted by $0.15 \pm 0.02 \AA$ toward lower values with respect to that of the simulated curves. A similar $\mathrm{C}-\mathrm{C}$ bond length contraction has been neither reported as result of any electron diffraction measurement nor predicted by calculations of stability of CNTs with diameter greater than $1 \mathrm{~nm} .{ }^{39-41}$ In previous work we interpreted the observed reduction of the first $F(R)$ peak in terms of the presence of an asymmetric radial distribution function, $g(R)$, due to the lack of atoms in the direction perpendicular to the SWCNT surface. The asymmetric $g(R)$ cannot be ascribed to the static disorder affecting the carbon nanotubes hexagonal network, because in the case of amorphous carbon films no contraction of $\mathrm{C}-\mathrm{C}$ first coordination shell has been observed. ${ }^{22,24,25}$ Indeed, although carbon nanotubes and amorphous carbon (a-C) films are basically different in nature and properties, their local structure in terms of $\mathrm{C}-\mathrm{C}$ nearest-neighbor bonding is quite similar (i.e., the local structure of $\mathrm{a}-\mathrm{C}$ films consists of a chainlike network of double and single carbon bonds forming a statically and dynamically disordered random matrix), according to the work of Comelli et al. ${ }^{24}$ The most evident proof of the validity of this picture is the carbon $\mathrm{K}$ near-edge of the amorphous film, which mimics quite well that of graphite and not that of diamond. This means that locally (i.e., around the carbon atom) small changes in bond $\mathrm{C}-\mathrm{C}$ length, bond angle, or hybridization do not affect the symmetry of the $g(R)$. The bond length contraction of the nearest neighbor observed exclusively in the $F(R)$ of SWCNTs (no comparable shift was seen in HOPG or even in MWCNTs) demonstrates that another kind of effect, different from pure thermal disorder, is responsible for such particular behavior in SWCNTs. This kind of first-coordination-shell contraction (or expansion) can be evidenced only through EXAFS-like data because these measurements probe the high- $k$ region (i.e., the low- $R$ region in the radial space). Thus, contrary to diffraction data (that have never reported on this effect), EXAFS (or EXELFS) is particularly sensitive to any variation in the shape of the $g(R)$.

The first-coordination-shell contraction in SWCNTs was found to be slightly dependent on the number of tubes that form the bundles. This is illustrated in Figure 6 where the nearestneighbor positions in the $F(R)$ curves are compared for two bundles of parallel SWCNTs with two different diameters, namely, $22 \pm 2$ and $5 \pm 1 \mathrm{~nm}$ (formed by a maximum of 340 and 18 nanotubes, respectively). The TEM image of this latter bundle is shown in the inset of Figure 6. In fact, the shift of the $F(R)$ peak is of about $0.03 \pm 0.02 \AA$ toward lower $R$ values,

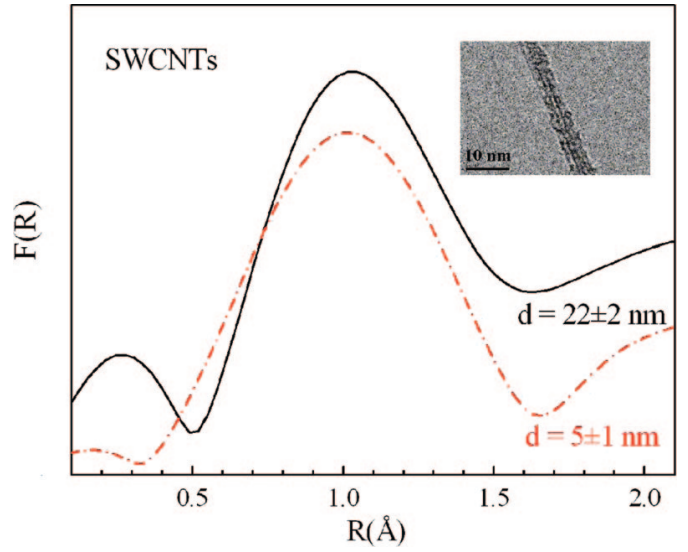

Figure 6. Fourier transform curves for two bundle of parallel SWCNTs with two different diameters $(d): d=22 \pm 2 \mathrm{~nm}$ (solid black line) and $d=5 \pm 1 \mathrm{~nm}$ (dot-dashed red line). In the inset, the high-resolution TEM images of the latter SWCNT bundle investigated by EXELFS. The bundles are seen to be formed by parallel alignment of SWCNTs.

when passing from a bundle of $\sim 340$ tubes to the $\sim 18$ tubes one. This suggests that a considerable reduction of the number of nanotubes in the bundle only slightly decreases the reported apparent contraction. In other words, the radial motion of the $\mathrm{C}$ atoms in SWCNTs seems to be slightly affected by the number of the tubes in the rope. Ultimately, such an anharmonic effect could be slightly enhanced and eventually reaches its maximum in a single tube.

As this anharmonic effect is not accounted for in the theoretical model that simulates the EXELFS spectra (the calculated $\chi(k)$ signals account for dynamical atomic motion only through the Debye-Waller factor), it is thus not surprising to observe such a discrepancy (position shift of the first neighbor) between the experimental and simulated data. In this work, to account for this anharmonic effect, we estimate the degree of asymmetry of the $g(R)$ for HOPG and SWCNTs along the lines of a simple model using the following formula to calculate the contribution of the first nearest neighbors to the $\chi(k)$ signal: ${ }^{42,43}$

$$
\chi_{1}(k)=\int g(R) \mathrm{e}^{-2 R / \lambda} \frac{A_{1}(k, \pi)}{k R^{2}} \sin \left(2 k R_{1}+\Phi_{1}(k)\right) \mathrm{d} R
$$

where the scattering amplitude $A_{1}(k, \pi)$ and the phase shift $\Phi_{1}(k)$ of the carbon atom first nearest neighbors are extracted from the experimental HOPG spectrum. We used the same $A_{1}(k, \pi)$ and $\Phi_{1}(k)$ values for SWCNTs, since the simulated ones result to assume the same values in all the $k$ wavevector range thus establishing the complete amplitude and phase transferability of the $\mathrm{C}-\mathrm{C}$ EXELFS signal for carbon atoms in slightly different structures. The value of $R_{1}$ has been chosen to be 1.42 $\AA$. The mean free path $\lambda$ has been considered to have a linear dependence on the wavevector $k,{ }^{44}$ and the pair distribution function $g(R)$ to be

$$
g(R)=\mathrm{e}^{-U(R) / k_{\mathrm{B}} T}
$$

where $U(R)$ is assumed to be a Morse potential of the form: ${ }^{45,46}$

$$
U(R)=D\left(\mathrm{e}^{-2 \alpha\left(R-R_{1}\right)}-2 \mathrm{e}^{-\alpha\left(R-R_{1}\right)}\right)
$$

where the binding energy, $D$, was considered to be of $1 \mathrm{eV} /$ atom and the measurements were performed at room temperature. Through an iterative process we evaluated the values for the $\alpha$ parameter giving rise to the Fourier transforms that best reproduce the experimental data recorded for both the bundles. 


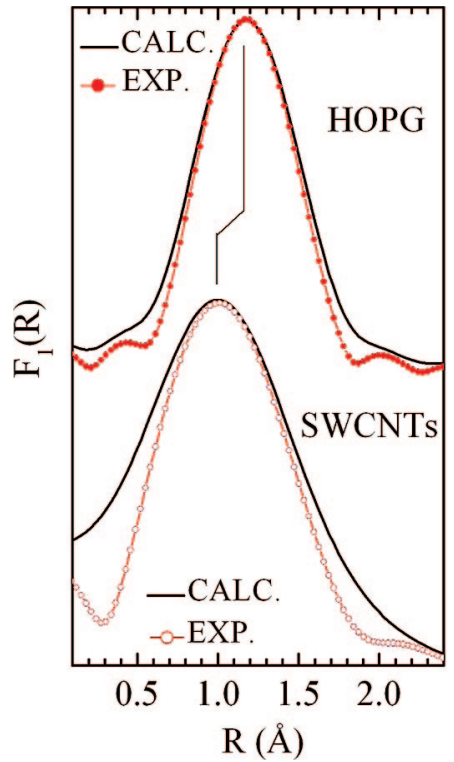

Figure 7. Fourier transforms of the experimental $k \chi_{1}(k)$ compared to those calculated taking into account the asymmetry of the $g(R)$ function. The best fit is obtained for $\alpha=2 \AA^{-1}$ and $\alpha=0.62 \AA^{-1}$ for HOPG and SWCNT bundle of $22 \mathrm{~nm}$ diameter, respectively.

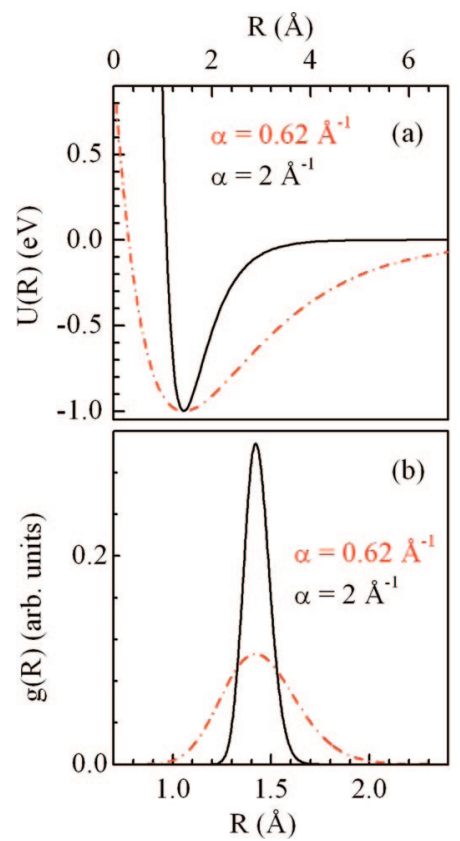

Figure 8. Comparison of Morse potential (a) and $g(R)$ function (b) calculated for two different $\alpha$ values: $\alpha=2 \AA^{-1}$ (solid black curves) corresponding to HOPG and $\alpha=0.62 \AA^{-1}$ (dot-dashed red curves) corresponding to the $22 \mathrm{~nm}$ diameter bundle of SWCNTs.

We found that $\alpha$ for graphite is $2.00 \pm 0.01 \AA^{-1}$, whereas for the bundle of SWCNTs of 22 and $5 \mathrm{~nm}$ diameter it is $0.62 \pm$ $0.01 \AA^{-1}$ and $0.60 \pm 0.01 \AA^{-1}$, respectively. Figure 7 displays the comparison between the experimental and simulated $F_{1}(R)$ curves for HOPG and the $22 \mathrm{~nm}$ diameter bundle, showing that a perfect fit is obtained. Parts a and b of Figure 8 show the respective potential $U(R)$ and the corresponding $g(R)$ used to obtain the result of Figure 7. The $\alpha$ value for graphite is high enough to make the $g(R)$ curve symmetric, whereas the latter value (for SWCNTs) is necessary to shift the $F_{1}(R)$ peak toward lower $R$ values. As mentioned above, information on the anharmonicity of carbon atoms on the SWCNT surface is expected to be at its maximum by recording an EXELFS spectrum on an isolated tube. Nevertheless, as the $\alpha$ value was found to be quite the same for both bundle diameters (passing from 340 to 18 nanotubes), we expect that the $\alpha$ value will be only slightly lower than the value estimated here, passing from a bundle of several tubes to a single tube.

In conclusion, we have measured the EXELFS oscillations above the ionization carbon $\mathrm{K}$ edge in the energy loss spectra by using a TEM apparatus that allowed us to acquire selectively EXELFS signals with nanoscale spatial resolution. The excellent agreement between the EXAFS and EXELFS data for HOPG confirmed that we are within the legitimacy of the dipole approximation and validated our EXAFS-like analysis. To theoretically reconstruct the EXELFS spectra with all the fine details of the oscillations over a wide $k$ range of $3-11 \AA^{-1}$, we found that we have to consider both (i) a cluster radius around the ionization carbon atom of $8 \AA$ and (ii) a full multiple scattering approach with the contribution of all the $n_{\mathrm{b}}$-body paths with $n_{\mathrm{b}}$ of up to 8 . This technique is shown to be extremely powerful to determine the chiral vector of the nanotubes because different $(n, m)$ values were observed to give rise to different $\chi(k)$ signals. This approach opens the possibility to determine straightforwardly the chirality of a single nanotube through EXELFS characterization. In the present study, we did not succeed in recording EXELFS spectra on individual nanotubes because of the long exposure time to the electron beam. Under our present TEM conditions, we succeeded in acquiring EXELFS spectra on bundles with a minimum diameter of $5 \mathrm{~nm}$. Finally, the asymmetry of the $g(R)$ induced by the higher outof-plane motion of the carbon atoms in SWCNTs has been evaluated by using a relatively simple model and demonstrating that this asymmetry is sizable compared to that of graphite. This anharmonic effect evidenced here explains the Raman behavior of SWCNTs recorded as a function of temperature.

Acknowledgment. This work has been conducted in the framework of a collaborative project financially supported by the Italian Ministry of foreign affairs and the MDEIE Ministry of Québec. M.A.E. and F.R. are also supported by FQRNT of Québec and NSERC of Canada. F.R. is grateful to the Canada Research Chairs program for partial salary support.

\section{References and Notes}

(1) Oberlin, A.; Endo, M.; Koyama, T. J. Cryst. Growth 1976, 32, 335.

(2) Iijima, S. Nature (London) 1991, 354, 56.

(3) Saito, R.; Dresselhaus, G.; Dresselhaus, M. S. Physical Properties of Carbon Nanotubes; Imperial College Press: London, 1998; Chapter 4.

(4) Wildoer, J. W. G.; Venema, L. C.; Rinzler, A. G.; Smalley, R. E.; Dekker, C. Nature (London) 1998, 391, 59.

(5) Odom, T. W.; Huang, J.-L.; Kim, P.; Lieber, C. M. Nature (London) 1998, $391,62$.

(6) Iijima, S.; Ichihashi, T. Nature (London) 1993, 363, 603.

(7) Qin, L.-C. In Progress in Transmission Electron Microscopy 2, Applications in Materials Science; Zhang, X. F., Zhang, Z., Eds.; Springer Series in Surface Sciences, Vol. 39; Springer-Verlag/TUP: Berlin, Heidelberg, Germany, 2001; pp 73-104.

(8) Meyer, J. C.; Paillet, M.; Duesberg, G. S.; Roth, S. Ultramicroscopy 2006, 106, 176, and references therein.

(9) Castrucci, P.; Tombolini, F.; Scarselli, M.; Bini, S.; De Crescenzi, M.; Diociaiuti, M.; Casciardi, S.; El Khakani, M. A.; Rosei, F. Phys. Rev. $B$ 2007, 75, 035420 .

(10) Huong, P. V.; Cavagnat, R.; Ajayan, P. M.; Stephan, O. Phys. Rev. B 1995, 51, 10048.

(11) Li, H. D.; Yue, K. T.; Lian, Z. L.; Zhan, Y.; Zhou, L. X.; Zhang, S. L.; Shi, Z. J.; Gu, Z. N.; Liu, B. B.; Yang, R. S.; Yang, H. B.; Zou, G. T.; Zhang, Y.; Iijima, S. Appl. Phys. Lett. 2000, 76, 2053, and references therein.

(12) Braidy, N.; El Khakani, M. A.; Botton, G. A. Carbon 2002, 40, 2835.

(13) De Crescenzi, M. Surf. Sci. Rep. 1995, 21, 89. 
(14) Castrucci, P.; Scarselli, M.; De Crescenzi, M.; Diociaiuti, M.; Chistolini, P.; El Khakani, M. A.; Rosei, F. Appl. Phys. Lett. 2005, 87, 103106.

(15) (a) Colliex, C. In Advances in Optical and Electron Microscopy; Barer, R., Cosslett, V. E., Eds.; Academic Press: London and New York, 1984; Vol. 9, p 161. (b) Colliex, C. Microsc., Microanal., Microsctruct. 1991, 2, 403.

(16) Lee, P. A.; Citrin, P. M.; Esenberger, P.; Kincaid, B. M. Rev. Mod. Phys. 1981, 53, 769.

(17) (a) For reviews of EXAFS spectroscopy, see: EXAFS Spectroscopy, Techniques, and Applications; Teo, B. K., Joy, D. C., Eds.; Plenum Press: New York, 1981. (b) Teo, B. K. EXAFS: Basic Principles and Data Analysis; Inorganic Chemistry Concepts 9; Springer-Verlag: Heidelberg, Germany, 1986.

(18) Stöhr, J. In X-ray Absorption: Principles, Applications, Techniques of EXAFS, SEXAFS and XANES; Koningsberger, D. C., Prins, R., Eds.; J. Wiley and Sons: New York, 1988; Vol. 92, p 443.

(19) Ritsko, J. J.; Gibbons, P. C.; Schnatterly, S. E. Phys. Rev. Lett. 1974, 37, 1068.

(20) This contrasts with the EXAFS experiment where the exciting radiation $(\hbar \omega)$ selects the transition between the fundamental and excited states, imposing thereby a continuous variation of the photon energy to be able to measure the onset of the nonoccupied states above the Fermi level.

(21) Egerton, R. F. In Electron Energy-Loss Spectroscopy in the Electron Microscope; Plenum Press: New York, 1996.

(22) Denley, D.; Perfetti, P.; Williams, R. S.; Shirley, D. A.; Stöhr, J. Phys. Rev. B 1980, 21, 2267.

(23) Comelli, G.; Stöhr, J.; Jark, W.; Pate, B. B. Phys. Rev. B 1988, 37, 4383.

(24) Comelli, G.; Stöhr, J.; Robinson, C. J.; Jark, W. Phys. Rev. B 1988, 38,7511 .

(25) Diaz, J.; Monteiro, O. R.; Hussain, Z. Phys. Rev. B 2007, 76, 094201 .

(26) Kincaid, B. M.; Meixmer, A. E.; Platzman, P. M. Phys. Rev. Lett. 1978, 40, 1296.

(27) Disko, M. M.; Krivanek, O. L.; Rez, T. Phys. Rev. B 1982, 25, 4252 .

(28) Landau, L.; Lifschitz, E. In Mécanique Quantique, Théorie non Relativiste, 3rd ed.; MIR: Moscow, 1974.
(29) Leapmann, R. D.; Rez, P.; Mayers, D. F. J. Chem. Phys. 1980, 72, 1232

(30) Leapmann, R. D.; Grunes, L. A.; Fejes, P. L. Phys. Rev. B 1982, $26,614$.

(31) (a) Ankudinov, A. L.; Ravel, B.; Rehr, J. J.; Conradson, S. D. Phys. Rev. $B$ 1998, 58, 7565. (b) Ankudinov, A. L.; Bouldin, C.; Rehr, J. J.; Sims, J.; Hung, H. Phys. Rev. B 2002, 65, 104107.

(32) Mattheiss, L. F. Phys. Rev. 1964, 133, A1399.

(33) Hedin, L.; Lundqvist, B. I. J. Phys. C: Solid State Phys. 1971, 4, 2064.

(34) Chen, R.; Trucano, P. Acta Crystallogr., Sect. A 1978, 34, 979.

(35) Pettifer, R. F.; Brouder, C.; Benfatto, M.; Natoli, C. R.; Hermes, C.; Ruiz López, M. F. Phys. Rev. B 1990, 42, 37.

(36) All $n_{\mathrm{b}}$-body paths contributions means, for example, that in the $n_{\mathrm{b}}$ $=2$ spectrum of Figure $2 \mathrm{a}$ are included all the single backscattering paths one can find inside the given radius of the cluster. In the $n_{\mathrm{b}}=3$ spectrum there are all the single scattering contributions plus all the triple scattering paths, and so on. The complexity of paths increases with $n_{\mathrm{b}}$. All the contributions from all the $n_{\mathrm{b}}$-body configuration paths should be summed up to generate the actual experimental EXELFS spectrum. The contribution of complex paths is found to be even essential for short interatomic distances $\left(k \leq 4 \AA^{-1}\right)$.

(37) Wyckoff, R. W. G. In Crystal Structures; John Wiley \& Sons: New York, London, 1963; Vol. I, p 26.

(38) https://www.ccs.uky.edu/ ernst/carbontubes/TubeApplet.html. (Accessed March and May 2008).

(39) Lambin, Ph.; Meunier, V.; Henrard, L.; Lucas, A. A. Carbon 2000, 38,1713 .

(40) Hirahara, K.; Bandow, S.; Kataura, H.; Kociak, M.; Iijima, S. Phys. Rev. B 2004, 70, 205422.

(41) Peralta-Inga, Z.; Boyd, S.; Murray, J. S.; O'Connor, C. J.; Politzer, P. Struct. Chem. 2003, 14, 431.

(42) Eisenberger, P.; Brown, G. S. Solid State Commun. 1979, $29,481$.

(43) De Crescenzi, M.; Antonangeli, F.; Bellini, C.; Rosei, R. Solid State Commun. 1983, 46, 875.

(44) Seah, M. P.; Dench, W. A. Surf. Interface Anal. 1979, 1, 2.

(45) Jackson, D. P. Surf. Sci. 1974, 43, 431.

(46) Girifalco, L. A.; Weizer, V. G. Phys. Rev. 1959, 114, 687.

JP810936C 